\title{
Rapamycin Added to Diet in Late Mid-Life Delays Age-Related Hearing Loss in UMHET4 Mice
}

\author{
Richard A. Altschuler ${ }^{1,2,3 *}$, Lisa Kabara ${ }^{1}$, Catherine Martin ${ }^{1}$, Ariane Kanicki ${ }^{1}$, \\ Courtney E. Stewart ${ }^{1,2}$, David C. Kohrman ${ }^{1}$ and David F. Dolan ${ }^{1}$ \\ ${ }^{1}$ Kresge Hearing Research Institute, Department of Otolaryngology, Head and Neck Surgery, University of Michigan, Ann \\ Arbor, MI, United States, ${ }^{2}$ VA Ann Arbor Health Care System, Ann Arbor, MI, United States, ${ }^{3}$ Department of Cell and \\ Developmental Biology, University of Michigan, Ann Arbor, MI, United States
}

Our previous study demonstrated rapamycin added to diet at 4 months of age had significantly less age-related outer hair cell loss in the basal half of the cochlea at 22 months of age compared to mice without rapamycin. The present study tested adding rapamycin to diet later in life, at 14 months of age, and added a longitudinal assessment of auditory brain stem response (ABR). The present study used UMHET4 mice, a 4 way cross in which all grandparental strains lack the Cdh23753A allele that predisposes to early onset, progressive hearing loss. UMHET4 mice typically have normal hearing until

OPEN ACCESS

Edited by:

Isabel Varela-Nieto,

Consejo Superior de Investigaciones

Cientificas (CSIC), Spain

Reviewed by:

Norio Yamamoto,

Kyoto University Hospital, Japan

Nesrine Benkafadar,

Stanford University, United States

*Correspondence:

Richard A. Altschuler

shuler@umich.edu

Specialty section:

This article was submitted to

Cellular Neuropathology,

a section of the journal

Frontiers in Cellular Neuroscience

Received: 26 January 2021

Accepted: 11 March 2021

Published: 07 April 2021

Citation:

Altschuler RA, Kabara L, Martin C, Kanicki A, Stewart CE, Kohrman DC and Dolan DF (2021) Rapamycin Added to Diet in Late Mid-Life Delays Age-Related Hearing Loss in UMHET4

Front. Cell. Neurosci. 15:658972. doi: 10.3389/fncel.2021.658972 16-17 months, then exhibit threshold shifts at low frequencies/apical cochlea and later in more basal high frequency regions. ABR thresholds at $4,12,24$, and $48 \mathrm{kHz}$ were assessed at 12, 18, and 24 months of age and compared to baseline ABR thresholds acquired at 5 months of age to determine threshold shifts (TS). There was no TS at 12 months of age at any frequency tested. At 18 months of age mice with rapamycin added to diet at 14 months had a significantly lower mean TS at 4 and $12 \mathrm{kHz}$ compared to mice on control diet with no significant difference at 24 and $48 \mathrm{kHz}$. At 24 months of age, the mean $4 \mathrm{kHz}$ TS in rapamycin diet group was no longer significantly lower than the control diet group, while the $12 \mathrm{kHz}$ mean remained significantly lower. Mean TS at 24 and $48 \mathrm{kHz}$ in the rapamycin diet group became significantly lower than in the control diet group at 24 months. Hair cell counts at 24 months showed large loss in the apical half of most rapamycin and control diet mice cochleae with no significant difference between groups. There was only mild outer hair cell loss in the basal half of rapamycin and control diet mice cochleae with no significant difference between groups. The results show that a later life addition of rapamycin can decrease age-related hearing loss in the mouse model, however, it also suggests that this decrease is a delay/deceleration rather than a complete prevention.

Keywords: rapamycin, age-related hearing loss, aging, auditory, cochlea, deafness

\section{INTRODUCTION}

Age-related hearing loss (ARHL) occurs in approximately one-third of people in the United States over the age of 65 increasing to approximately half of those over the age of 75 (e.g., Gates, 2006; Gates et al., 2010). ARHL can reduce ability to communicate, quality of life and social integration and has been identified as a major risk factor for depression and dementia 
(e.g., Gates et al., 2010; Lin et al., 2011; Davis and Smith, 2013). One major cause of ARHL is an age-related loss of sensory hair cells, predominantly outer hair cells, and an accompanying decrease in auditory sensitivity as measured by threshold shifts (TS) in auditory brain stem response (ABR) in animal models and audiometric thresholds in people. The underlying mechanisms responsible for age-related hair cell loss remain unknown and there are no treatments currently being clinically applied to prevent or reduce this pathology.

The National Institute on Aging Intervention Testing Program (NIA-ITP) tests for treatments that can be added to diet to increase lifespan, using UMHET3 mice, a four-way cross, as a model. Four-way cross mice (from four different grandparent strains) provide for genetic heterogeneity and reduce strain specific effects. Among several effective treatments identified through NIA-ITP, addition of rapamycin to diet at 9 months of age was found to increase life span by $26 \%$ in male mice and $23 \%$ in female mice (Miller et al., 2014). We hypothesized that ARHL might share underlying mechanisms, such that treatments that enhance life span could also reduce and/or delay ARHL. This is consistent with studies that demonstrate the positive effects of rapamycin on age-related disorders in animal models, including decreases in cardiac pathology (Dai et al., 2014); muscle weakness (Bitto et al., 2016), cancer incidence (Anisimov et al., 2011), and cognitive decline (Halloran Hussong et al., 2012; Majumder et al., 2012).

In a previous study (Altschuler et al., 2018) we evaluated cochleae from 22 months old UMHET3 mice that had rapamycin added to their diet at 4 months of age as well as from control littermates with normal diet. The 22 months old rapamycintreated mice had significantly less loss of outer hair cells in the basal half of the cochlea compared to the untreated controls. This sparing of hair cell loss was restricted to the basal half of the cochleae, while the apical half of the cochleae exhibited equivalently large losses of outer hair cells in both rapamycin-fed and normal diet controls (Altschuler et al., 2018).

These results showing rapamycin could reduce or delay agerelated hair cell loss in the basal half of the cochlea at 22 months of age raised two pertinent issues. First, the apparent limitation of the treatment effect of rapamycin to the basal half of the cochlea could reflect differences in mechanisms underlying hair cell loss along the cochlear spiral. Alternatively, the effect of rapamycin treatment could be due to delaying rather than preventing agerelated hair cell loss. Hair cell loss occurs earlier in apical vs. basal cochlea in most mouse strains (for reviews; Gratton and Vazquez, 2003; Ohlemiller, 2006). If the effect of rapamycin is to "delay" rather than prevent age-related hair cell loss, a treatment induced difference in apical cochleae might also have been present at an earlier time, but by 22 months of age the delay was over and the hair cell loss had equilibrated. The current study was designed to address these points by generating a longitudinal assessment of auditory brain stem response (ABR) thresholds at $4,12,24$, and $48 \mathrm{kHz}$ in individual mice at 5, 12, 18, and 24 months of age. The current study also addressed a second question of whether beginning rapamycin treatment at a more clinically relevant later age would still be effective in reducing or delaying ARHL. Recent studies have shown that rapamycin can extend life span in mice even when added to diet at 1920 months of age (Harrison et al., 2009; Zhang et al., 2014) and can also reduce age-related pathologies such as cancer incidence and decreased muscle (Zhang et al., 2014) and cardiac function (Quarles and Rabinovitch, 2020) with late life application. We therefore chose to add rapamycin at a later time but prior to the first appearance of ARHL. Three of the four "grandparent strains" of the UMHET3 mice carry homozygous ahl alleles $\left(C d h 23^{753 A}\right)$ that predispose to early onset, hair cell loss and progressive deafness in mice, thus restricting the progeny that can be used in auditory aging studies and decreasing the utility of this four way cross for auditory aging studies (Noben-Trauth et al., 2003; Mianné et al., 2016). For this reason, earlier studies from our group (Schacht et al., 2012; Altschuler et al., 2015) developed a different four-way cross, UMHET4, in which the grandparent strains lack the ahl predisposing Cdh23 $3^{753 \mathrm{~A}}$ alleles. We returned to use of UMHET4 mice in the current study so that all of the progeny could be used. Our previous studies using UMHET4 mice (Schacht et al., 2012; Altschuler et al., 2015) and pilot animals in the current study showed that UMHET4 mice generally have a later appearing ARHL than UMHET3 mice and that ABR TS do not commonly initiate until around 18 months of age. The current study therefore tested the influence of adding rapamycin to mouse diet at 14 months of age.

\section{STUDY DESIGN}

UMHET4 mice were entered into the study sequentially as they reached appropriate age points and tested for $A B R$ as they reached 5 months of age. Mice with $\mathrm{ABR}$ thresholds more than three standard deviations from the mean at any of the four frequencies $(4,12,24$, and $48 \mathrm{kHz})$ assessed were excluded from the study. The ABR thresholds at 5 months of age served as individual and group mean baselines and the basis for determining TS at later ages. Mice received a second ABR at 12 months of age and any mice with greater than $10 \mathrm{~dB}$ SPL TS at any frequency tested were excluded from the study. At 14 months of age, eligible male and female mice were randomly placed into one of two groups, either a rapamycin diet group or a control diet group. Rapamycin was given encapsulated in the food (pelleted chow) to the mice in the rapamycin treatment group at a dose of $42 \mathrm{mg} \mathrm{kg}^{-1}$, the dose found most effective in increasing lifespan by Miller et al. (2014). Both groups had diet changed at 14 months of age, from standard chow to either rapamycin diet or control diet without rapamycin (but containing the other additions to the rapamycin chow that allowed the rapamycin to be encapsulated). Longitudinal ABR measures from mice in both groups were continued at 18 and 24 months of age and TS (from 5 months old baseline) determined. The control diet group started with 37 mice with 29 surviving until euthanasia at 24 months of age and the rapamycin diet group started with 29 mice with 24 surviving until the 24 months old end point. Animals were euthanized within eight days after the 24 months old ABR measure. The left cochleae were used for hair cell count evaluations (cytocochleograms) and the right cochleae were processed and saved for future gene expression studies. 


\section{Breeding}

The UM-HET4 mice were generated as described previously (Schacht et al., 2012) by a four-way cross between MOLF/EiJ (Jackson Laboratory stock \#000550) × 129S1/SvImJ F1 (Jackson Laboratory stock \#002448) female mice and C3H/HeJ (Jackson Laboratory stock \#000659) $\times$ FVB/NJ F1 (Jackson Laboratory stock \#001800) male mice. All of the four grandparental strains lack the $a h l$ allele that typically leads to hearing loss appearing at 2-4 months of age (Johnson et al., 2006, for review). Each mouse in the tested UM-HET4 population inherits $25 \%$ of its genome from each of the four distinct inbred grandparental stocks and is genetically unique, but shares $50 \%$ of its alleles with every other mouse in the tested population. The UMHET4 mice exhibit variability in their ARHL hearing loss that was correlated with polymorphisms in specific genetic loci (Schacht et al., 2012).
Auditory Brain Stem Response (ABR)

Animals were anesthetized with ketamine $(65 \mathrm{mg} / \mathrm{kg})$, xylazine (7 mg/kg), and acepromazine (2 mg/kg). Body temperature was maintained with water circulating heating pads and heat lamps. Additional anesthetic (ketamine and xylazine) was administered when needed to maintain anesthesia depth sufficient to insure immobilization and relaxation. ABRs were then recorded in an electrically and acoustically shielded chamber (Acoustic Systems, Austin, TX, USA). Needle electrodes were placed at vertex (active), the test ear (reference) and the contralateral ear (ground) pinnae. Tucker Davis Technologies (TDT) System III hardware and SigGen/BioSig software (TDT, Alachua, FL, USA) were used to present the stimulus and record responses. Tones were delivered through a EC1 sound driver (TDT) with the speculum placed just inside the tragus. Stimulus presentation used $15 \mathrm{~ms}$ tone bursts, with $1 \mathrm{~ms}$ rise/fall times, presented 10 per second.
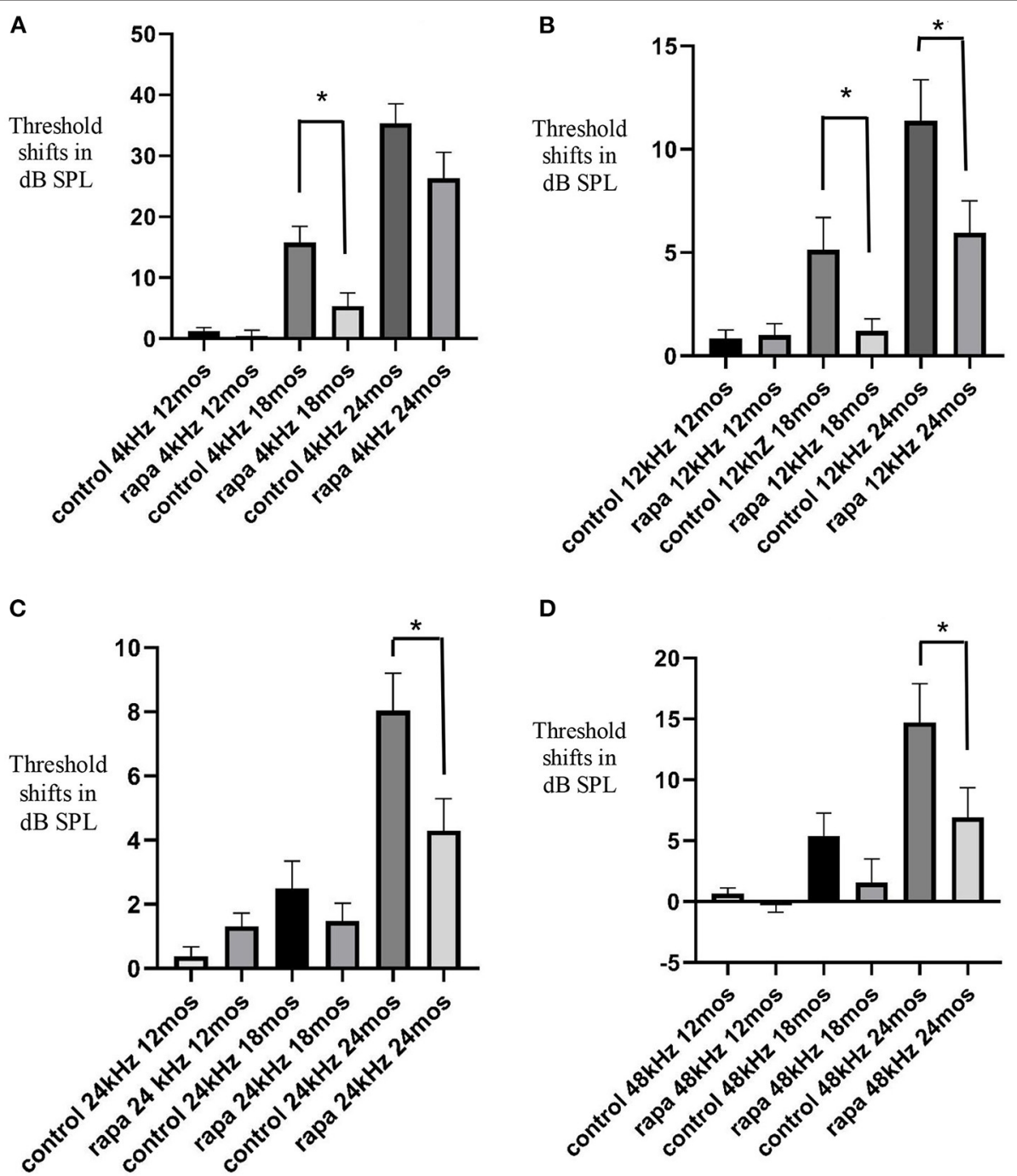

FIGURE 1 | Comparison of mean auditory brain stem response threshold shifts (compared to 5 months of age) in the group with rapamycin added to diet at 14 months of age (rapa) vs. the group on control diet without rapamycin (control) at 12, 18, and $24 \mathrm{months}$ of age at $4 \mathrm{kHz}$ (A), $12 \mathrm{kHz}$ (B), $24 \mathrm{kHz}$ (C) and $48 \mathrm{kHz}$ (D). Asterisks indicate significant differences. Please note differences between A, B, C, and D in scale bars for dB SPL on the "y" axis. 
Up to 1,024 responses were averaged for each stimulus level. Responses were collected for stimulus levels in $10 \mathrm{~dB}$ steps at higher stimulus levels, with additional $5 \mathrm{~dB}$ steps near threshold. Thresholds were interpolated between the lowest stimulus level where a response is observed, and $5 \mathrm{~dB}$ lower, where no response is observed. The frequencies tested were $4,12,24$, and $48 \mathrm{kHz}$.

\section{Histology}

Mice were euthanized by intraperitoneal injection of Sodium Pentobarbital (Fatal Plus) followed by decapitation. Cochleae were rapidly removed and middle ears assessed for signs of middle ear infection under a dissection microscope. An opening made through the otic capsule in the apex of the left cochleae and fixative (4\% paraformaldehyde in phosphate buffer) was slowly infused into the cochlear fluids. The cochleae were then immersed in fixative for $2-6 \mathrm{~h}$ at room temperature on a rotator and rinsed in phosphate buffered saline (PBS) before a 16$24 \mathrm{~h}$ decalcification in 5\% EDTA at room temperature. This was followed by removal of the otic capsule and tectorial membrane. Cochleae were then stained with 1\% Phalloidin- Alexa Fluor 568 in PBS and then micro dissected into three segments, apex, base, and hook. Each segment was mounted separately as a surface preparation on a glass slide with Prolong Gold as mounting media. Slides were stored at $4^{\circ} \mathrm{C}$ before examination and viewing.

\section{Hair Cell Assessment}

Phalloidin labeling of hair cells was used to identify presence or absence of hair cells. Hair cells were counted under epifluorescence optics on a Leica fluorescent microscope under double blind conditions. The number of inner hair cells and outer hair cells (OHC) that were present or absent for each $0.19 \mathrm{~mm}$ reticule length was entered into a cytocochleogram program starting at the apex and moving basally until the entire length of the cochlear spiral had been assessed. The program compares hair cell numbers to a normal data base. The program can generate a graph of hair cell loss by position along the cochlear spiral for each cochlea (cytocochleogram), can provide the analysis in absolute numbers, as the total percent of hair cells lost in each animal assessed and can be used to generate means for groups.

\section{Statistics}

Significance for ABR threshold changes was tested using an unpaired " $\mathrm{t}$ " test with Welch's correction and the non-parametric Mann-Whitney test with Bonferroni adjustment. Significance was considered at both $p<0.01$ and $p<0.05$ levels. There was no significant difference in the mean ABR thresholds or TS between male and females within the rapamycin diet or within the diet control group at any age and so males and females were combined when testing for significance. Means are given in text plus/minus the standard error of the mean (SEM).

\section{RESULTS}

\section{Auditory Brain Stem Response}

There was no significant difference in the mean ABR thresholds or TS between male and females in either control diet or rapamycin diet groups at any age for any of the frequencies

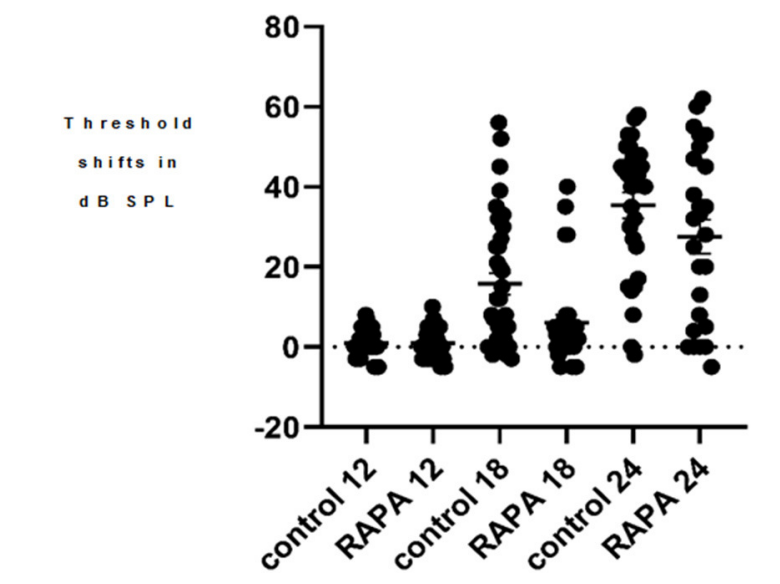

FIGURE 2 | A scatter plot of the $4 \mathrm{kHz}$ auditory brain stem response threshold shifts (compared to 5 months of age) at 12, 18, and 24 months of age in the control diet group (without rapamycin) and in the group with rapamycin added to diet at 14 months of age. There is little variability in either group at 12 months of age. At 18 months of age more variability is seen in the control diet group (control 18) while the rapamycin treatment group (RAPA18) remains more skewed toward little threshold shift. At 24 months of age there is less variability in the control group (control 24) now skewed toward large threshold shifts while the rapamycin treatment group (RAPA24) has more distribution across the range of threshold shifts.

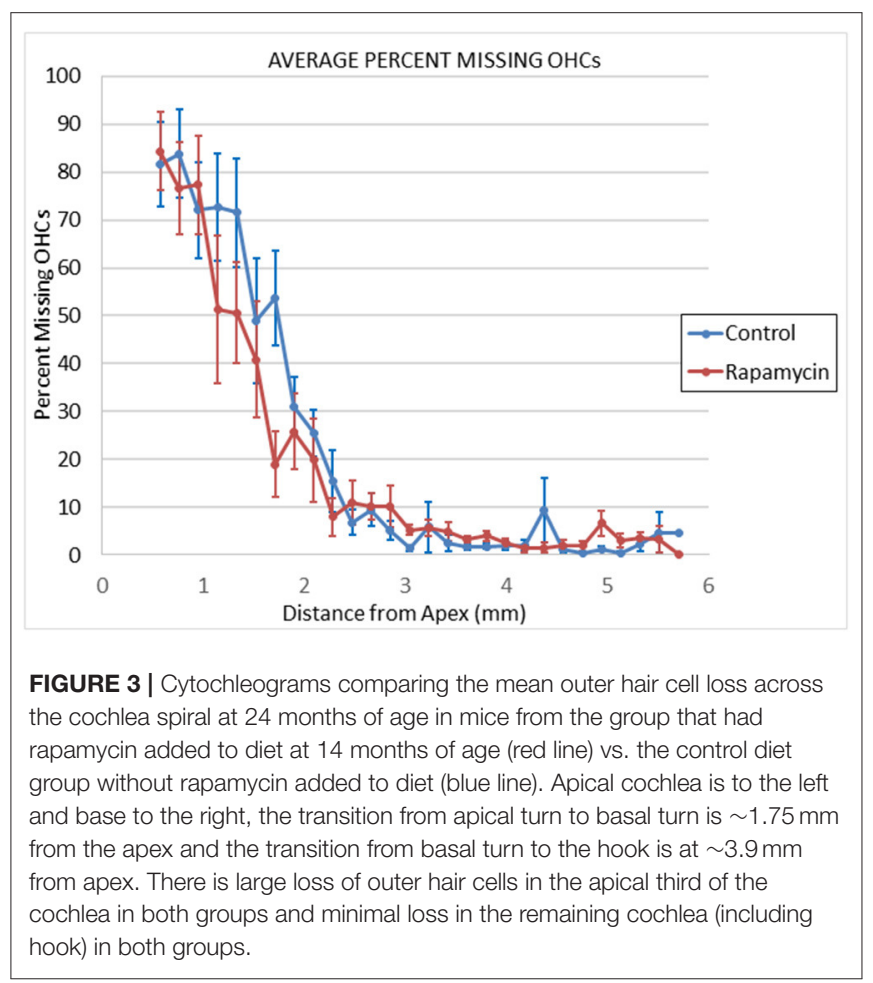

assessed and males and females were therefore combined for comparisons. At 12 months of age there were no animals with TS above $10 \mathrm{~dB}$ SPL at any frequency in animals to be assigned to either group. The progression of ABR TS in the control diet group 

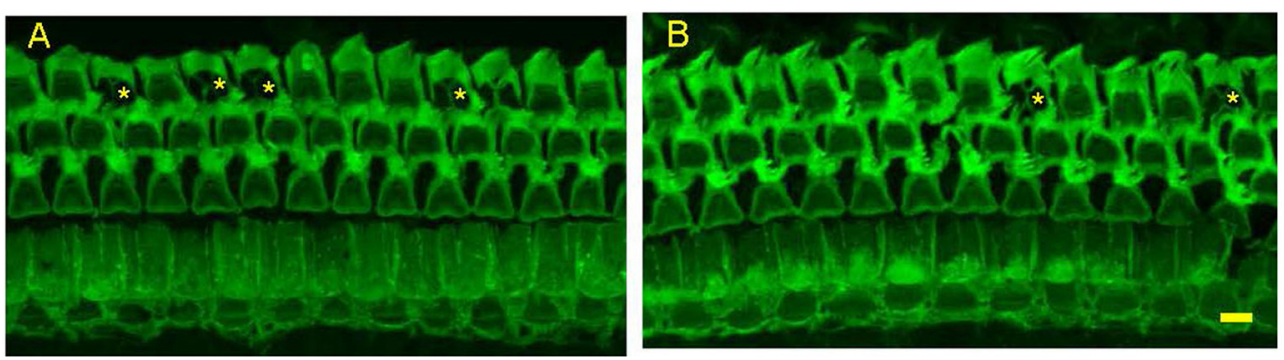

FIGURE 4 | Representative photomicrographs from surface preparations of organ of Corti from a 24 months old Control Diet mouse (A) and a 24 months old mouse on the Rapamycin Diet $\mathbf{B})$ Both have outer hair cell loss in the third row, with scars marked by asterisks. Bar $=10 \mu$.

was consistent with the pattern we have previously reported in UMHET4 mice with significant TS first appearing at low frequencies and later at higher frequencies (Schacht et al., 2012; Altschuler et al., 2015) (Figures 1A-D). There was variability across animals in the timing of their progression of hearing loss (e.g., Figure 2 for $4 \mathrm{kHz}$ ) with a few mice (3-29) in the control diet group retaining normal hearing thresholds across all frequencies even at the latest 24 months of age ABR assessment time, also consistent with what we have previously observed in UMHET4 mice (Schacht et al., 2012; Altschuler et al., 2015).

\section{Threshold Shifts at 18 Months of Age}

At 18 months of age, the mean ABR TS at $4 \mathrm{kHz}$ in the rapamycin diet group was $5.5 \pm 2.2 \mathrm{~dB}$ SPL compared to a $15.8 \pm 2.7 \mathrm{~dB}$ SPL mean in the control diet group, this difference was significant $(p<0.01)$ (Figure 1A). This was a consequence of a larger number of animals (17-37) showing $4 \mathrm{kHz}$ ABR TS over $10 \mathrm{~dB}$ in the control diet group compared to 5-29 in the rapamycin diet group. Variability among mice in timing of TS and rapamycin treatment effect is shown in a scatter plot at $4 \mathrm{kHz}$ in Figure 2. At $12 \mathrm{kHz}$ the mean TS in the rapamycin diet group was 1.2 $\pm 0.6 \mathrm{~dB}$ SPL compared to a $5.1 \pm 1 / 6 \mathrm{~dB}$ SPL in the control diet group, this difference was also significant $(p<0.05)$. There were no significant differences in 24 or $48 \mathrm{kHz}$ TS means between rapamycin and control diet groups (Figures 1C,D). One mouse in the rapamycin diet group was an outlier with a large (53 dB SPL) TS at $48 \mathrm{kHz}$ without a TS at $4 \mathrm{kHz}$.

\section{Threshold Shifts at 24 Months of Age}

At 24 months of age there was no longer a significant difference $(p=01.4)$ in the mean $4 \mathrm{kHz}$ TS between the rapamycin diet $(27.2 \pm 4.5 \mathrm{~dB}$ SPL $)$ and the control diet $(35.3 \pm 3.2 \mathrm{~dB}$ SPL $)$ groups (Figure 1A). The difference in mean $12 \mathrm{kHz}$ TS between rapamycin diet $(5.9 \pm 1.5 \mathrm{~dB}$ SPL $)$ and control diet $(11.4 \pm 2.0$ $\mathrm{dB}$ SPL) groups remained significant $(p<0.05)$ (Figure 1B). Differences now appeared at higher frequencies, with a significant difference $(p<0.05)$ in mean TS between rapamycin and control diet groups for both $24 \mathrm{kHz}$ (rapamycin $=4.2 \pm 1.0 \mathrm{~dB}$ SPL; control $=8.0 \pm 1.2 \mathrm{~dB} \mathrm{SPL}$ ) and $48 \mathrm{kHz}$ (rapamycin $=6.9$ $\pm 2.5 \mathrm{~dB}$ SPL; control $=14.7 \pm 3.2 \mathrm{~dB}$ SPL) assessments (Figures 1C,D).

\section{Histology and Hair Cell Assessment}

There were no signs of middle ear infection in the cochleae of any mice in the study. At 24 months of age there was large outer hair cell loss in the apical half of cochlea of most mice in both control diet mice and rapamycin diet groups (Figure 4), with no significant difference in the mean loss between these groups. There was only minimal outer hair cell loss in the basal half of the cochlea of most mice in both groups with mean outer hair cell loss well under $10 \%$ in both groups (Figures 3, 4) despite ABR threshold shifts.

\section{DISCUSSION}

The results of the present study extend results of our previous study (Altschuler et al., 2018) that found rapamycin added to diet at 4 months of age reduced outer hair cell loss in the basal half but not apical half of the cochleae of 22 months old UMHET3 mice. The present study used longitudinal measure of $\mathrm{ABR}$ to show rapamycin added to diet reduced mean threshold shifts at 4 and $12 \mathrm{kHz}$ in 18 months old UMHET4 mice, largely by reducing the percent of mice showing TS. It is not well understood why hair cell loss occurs first in apical regions, then in basal regions in most mouse models and it has been suggested that different mechanisms could be influencing basal vs. apical age-related hearing loss (e.g., Schulte and Schmiedt, 1992; Dubno et al., 2013; Wu et al., 2020). Since $4 \mathrm{kHz}$ is processed in the apical half of the mouse cochlea, $\sim 1.25 \mathrm{~mm}$ from apex (Viberg and Canlon, 2004), the results of the present study show that the influence of rapamycin is not restricted to the basal half of the cochlea. This provides indirect evidence that at least some components underlying agerelated hearing loss (those that can be influenced by rapamycin) are present in both basal and apical cochleae. Rapamycin acts on mammalian-target-of-rapamycin (mTOR) pathways (both mTORC1 and mTORC2). These pathways are multifaceted and in turn act on other functional signaling pathways including those associated with metabolism, proliferation, immune response and cell survival (Inoki et al., 2005a,b; Perl, 2015 for reviews; Wataya-Kaneda, 2015). The mTORC1 pathway can influence endoplasmic reticulum (ER) stress and the unfolded protein response (UPR) (e.g., Ye et al., 2015). ER stress-related factors have been shown to increase in the cochleae of aged mice 
(Wang et al., 2015) and ER stress-mediated apoptosis has been associated with noise-induced, ototoxic drug-induced and age-related hearing losses (Oishi et al., 2015; Wang et al., 2015; Hu et al., 2016; Mahdi et al., 2016). ER stress pathways could therefore be a target of the rapamycin effect on ARHL. Rapamycin could also stimulate the survival pathway of $\mathrm{p}$-Akt (S473) via mTOR2 signaling, including reducing mitochondrial stress. Rapamycin could also act through its influence on the inflammatory response or through inhibition of oxidative stress pathways previously implicated in hair cell pathology (Yamasoba et al., 2013, for review). Future studies will be necessary to identify the specific target or targets and pathways underlying the treatment effect seen in the current study.

Another important result is that the rapamycin treatmentrelated sparing of $4 \mathrm{kHz}$ hearing loss at 18 months is no longer present at 24 months of age and there is an associated large hair cell loss in the apical cochlea of most rapamycin diet and control diet mice at 24 months of age, with no difference between the groups. This suggests that rapamycin treatment delays but does not prevent hearing loss. It would be valuable to identify the specific mechanisms by which rapamycin delays hair cell loss, not only to increase understanding of general mechanisms underlying ARHL but to determine if the delay could be extended and even turned into prevention. The timing of the last ABR and terminal euthanasia in the present study was before large TS generally occurs at higher frequencies in the UMHET4 mouse model. A greater rapamycin induced sparing of hair cell loss and TS than observed in the current study might therefore be found at a later age when greater hair cell loss is occurring, as observed in the previous study in UMHET3 mice where ARHL occurs more rapidly. The lack of correlation between ABR TS and $\mathrm{OHC}$ loss in the more basal cochlea at 24 months of age is consistent with reports of age-related reduction or loss in OHC function with reduced distortion product otoacoustic emissions (DPOAE) appearing before age-related OHC loss (e.g., Syka, 2010, for review). One explanation is an age-related disruption of prestin in morphologically intact OHC (Chen et al., 2009; Syka, 2010). It would be valuable to examine the influence of rapamycin treatment on age-related decrements in DPOAE. The variability in the progression and extent of ARHL seen in the control diet UMHET4 mice may reflect their genetic diversity and we have previously shown this variability can be correlated with polymorphisms in specific genetic loci (Schacht et al., 2012). The variability seen in the treatment effect of rapamycin in

\section{REFERENCES}

Altschuler, R. A., Kanicki, A., Martin, C., Kohrman, D., and Miller, R. A. (2018). Rapamycin but not acarbose decreases age-related loss of outer hair cells in the mouse Cochlea, Hearing Reserarch 370, 11-15. doi: 10.1016/j.heares.2018.09.003

Altschuler, R. A., Zabezhinski, M. A., Popovich, I. G., Piskunova, T. S, Semenchenko, A. V., Tyndyk, M. L., et al. (2015). Age-related changes in auditory nerve-inner hair cell connections, hair cell numbers, auditory brain stem response and gap detection in UMHET4 mice, Neuroscience 292, 22-33. doi: 10.1016/j.neuroscience.2015. 01.068 the rapamycin diet group might also reflect UMHET4 genetic diversity and it would be interesting to identify such differences in future studies.

The present study also addressed the question of whether beginning rapamycin treatment later in life than the 4 months of age used in Altschuler et al. (2018) would be effective. The results show beginning treatment later in life, at 14 months of age is still effective. This is consistent with studies showing late life rapamycin delivery also enhances life span and delays/reduces age-related declines in cardiac, muscle and cognitive functions (Quarles and Rabinovitch, 2020, for review). The literature also suggests that late life intermittent administration of rapamycin and rapamycin-like compounds ("rapalogs") with less side-effects in people, can also increase life span and decrease age-related declines (Anisimov et al., 2011; Arriola Apelo et al., 2016; Shavlakadze et al., 2018; Quarles and Rabinovitch, 2020). It would therefore be valuable to test late-life intermittent treatment effects of rapamycin and rapalogs on ARHL.

\section{DATA AVAILABILITY STATEMENT}

The raw data supporting the conclusions of this article will be made available by the authors, without undue reservation.

\section{ETHICS STATEMENT}

The animal study was reviewed and approved by VAAAHS Institutional Animal Care and Use Committee.

\section{AUTHOR CONTRIBUTIONS}

RA, DD, and DK: contributed to the study in design: $L K, A K$, and CM: carrying out studies and measures: RA, DD, DK, CM, and CS: analysis of results and their impact. All authors contributed to the article and approved the submitted version.

\section{FUNDING}

These studies were supported by Department of Veterans Affairs Merit Grant 1I01RX002431. The views expressed do not necessarily reflect the official policies of the Department of Health and Human Services, nor does mention of trade names, commercial practices, or organizations imply endorsement by the U.S. Government. 
Chen, G.-D., Li, M., Tanaka, C., Bielefeld, E. C., Hu, B. H., Kermany, M. H., et al. (2009). Aging outer hair cells (OHC) in the Fischer 344 rat cochlea: function and morphology. Hear. Res. 248, 39-47. doi: 10.1016/j.heares.2008.11.010

Dai, D. F., Karunadharma, P. P., Chiao Y. A., Basisty, N., Crispin, D., and Hsieh, E. J., (2014). Altered proteome turnover and remodeling by short-term caloric restriction or rapamycin rejuvenate the aging heart. Aging Cell 13, 529-539. doi: 10.1111 acel.12203

Davis, A., and Smith, P. (2013). Adult hearing screening: health policy issues-what happens next. Am. J. Audiol. 22, 167-170. doi: 10.1044/1059-0889(2013/12-0062)

Dubno, J. R., Eckert, M. A., Lee, F.-S., Matthews, L. J., and Schmiedt, R. A. (2013). Classifying human audiometric phenotypes of age-related hearing loss from animal models. J. Assoc. Res. Otolaryngol. 14, 687-701. doi: 10.1007/s10162-013-0396-x

Gates, G. A. (2006). The effect of noise on cochlear aging. Ear Hear. 27:91. doi: 10.1097/01.aud.0000194512.51849.ab

Gates, G. A., Gibbons, L. E., McCurry, S. M., Crane, P. K., Feeney, M. P., and Larson, E. B. (2010). Executive dysfunction and presbycusis in older persons with and without memory loss and dementia. Cogn. Behav. Neurol. 23, 218-223. doi: 10.1097/WNN.0b013e3181d748d7

Gratton, M. A., and Vazquez, A. E. (2003). Age-related hearing loss: current research. Curr. Opin. Otolaryngol. Head Neck Surg. 11, 367-371. doi: 10.1097/00020840-200310000-00010

Halloran Hussong, S. A., Burbank, R., Podlutskaya, N., Fischer, K. E., and Sloane, L. B. (2012). Chronic inhibition of mammalian target of rapamycin by rapamycin modulates cognitive and non-cognitive components of behavior throughout lifespan in mice. Neuroscience 223:102-13. doi: 10.1016/j.neuroscience.2012.06.054

Harrison, D. E., Strong, R., Sharp, Z. D., James, F., Nelson, C. M. and Kevin, A. (2009). Rapamycin fed late in life extends lifespan in genetically heterogeneous mice. Nature 460, 392-395. doi: 10.1038/nature08221

Hu, J., Li, B., Apisa, L., Yu, H., Entenman, S., Xu, M., Stepanyan, R., et al. (2016). ER stress inhibitor attenuates hearing loss and hair cell death inCdh23erl/erlmutant mice. Cell Death Dis. 7:e2485doi: 10.1038/cddis.2016.386

Inoki, K., Corradetti, M. N., and Guan, K. L. (2005b). Dysregulation of the TSCmTOR pathway in human disease. Nat. Genet. 37, 19-24. doi: 10.1038/ng1494

Inoki, K., Ouyang, H., Li, Y., and Guan, K. L. (2005a). Signaling by target of rapamycin proteins in cell growth control. Microbiol. Mol. Bio.l Rev. 69, 79-100. doi: 10.1128/MMBR.69.1.79-100.2005

Johnson, K. R., Zheng, Q. Y., and Noben-Trauth, K. (2006). Strain background effects and genetic modifiers of hearing in mice. Brain Res.1091, 79-88. doi: 10.1016/j.brainres.2006.02.021

Lin, F. R., Ferrucci, L., Metter, E. J., An, Y., Zonderman, A. B., and Resnick, S. M. (2011). Hearing loss and cognition in the Baltimore Longitudinal Study of Aging. Neuropsychology. 25, 763-770. doi: 10.1037/a0024238

Mahdi, A. A., Rizvi, S. H. M., and Parveen, A. (2016). Role of endoplasmic reticulum stress and unfolded protein responses in health and diseases. Ind. J. Clin.l Biochem. IJCB 31, 127. doi: 10.1007/s12291-015-0502-4

Majumder, S., Caccamo, A., Medina, D. X., Benavides, A. D., Javors, M. A., Kraig, E, Strong, R., et al. (2012). Lifelong rapamycin administration ameliorates age-dependent cognitive deficits by reducing IL-1beta and enhancing NMDA signaling. Aging Cell 11, 326-335. doi: 10.1111/j.1474-9726.2011.00791.x

Mianné, J., Chessum, L, Kumar, S., Aguilar, C., Codner, G., Hutchison, M., et al. (2016). Correction of the auditory phenotype in C57BL/6N mice via CRISPR/Cas9-mediated homology directed repair Genome Medicine 8:16. doi: 10.1186/s13073-016-0273-4

Miller, R. A., Harrison, D. E., Astle, C. M., Fernandez, E., Flurkey, K., Han, M., et al. (2014). Rapamycin-mediated lifespan increase in mice is dose and sex dependent and metabolically distinct from dietary restriction. Aging Cell. 13, 468-477. doi: 10.1111/acel.12194
Noben-Trauth, K., Zheng, Q. Y., and Johnson, K. R. (2003). Association of cadherin 23 with polygenic inheritance and genetic modification of sensorineural hearing loss. Nat. Genet. 345, 21-23. doi: 10.1038/ng1226

Ohlemiller, K. K. (2006). Contributions of mouse models to understanding age- and noise-related hearing loss. Brain Res. 109, 189-102. doi: 10.1016/j.brainres.2006.03.017

Oishi, N., Duscha, S., Boukari, H., Meyer, M., Xie, J., Wei, G., et al. (2015). XBP1 mitigates aminoglycoside-induced endoplasmic reticulum stress and neuronal cell death. Cell Death Dis. 6:e1763. doi: 10.1038/cddis.2015.108

Perl, A. (2015). mTOR activation is a biomarker and a central pathway to autoimmune disorders, cancer, obesity, and aging. Ann N Y Acad Sci. 1346, 33-44. doi: 10.1111/nyas.12756

Quarles, E., and Rabinovitch, P. S. (2020). Transient and later-life rapamycin for healthspan extension, Aging 12, 4050-4051. doi: 10.18632/aging.102947

Schacht, J., Altschuler, R. A., Burke, D. T., Chen, S., Dolan, D., Galecki, A. T., et al. (2012). Alleles that modulate late life hearing in genetically heterogeneous mice. Neurobiol. Aging, 1842. 15-29. doi: 10.1016/j.neurobiolaging.2011.12.034

Schulte, B. A., and Schmiedt, R. A. (1992). Lateral wall Na,K-ATPase and endocochlear potentials decline with age in quiet-reared gerbils. Hear Res. 61, 35-46. doi: 10.1016/0378-5955(92)90034-K

Shavlakadze, T., Zhu, J., Wang, S., Zhou, W., Morin, B., Egerman, M. A. et al., (2018). Short-term low-dose mTORC1 inhibition in aged rats counter-regulates age-related gene changes and blocks age-related kidney pathology. J Gerontol A Biol Sci Med Sci. 73, 845-852. doi: 10.1093/gerona/glx249

Syka, J. (2010). The Fischer 344 rat as a model of presbycusis. Hear. Res. 264, 70-78. doi: 10.1016/j.heares.2009.11.003

Viberg, A., and Canlon, B. (2004). The guide to plotting a cytocochleogram, Hear Res. 197, 1-10. doi: 10.1016/j.heares.2004.04.016

Wang, W., Sun, Y., Chen, S., Zhou, X., Wu, X., Kong, W., and Kong, W. (2015). Impaired unfolded protein response in the degeneration of cochlea cells in a mouse model of age-related hearing loss. Exp. Gerontol, 70, 61-70. doi: 10.1016/j.exger.2015.07.003

Wataya-Kaneda, M. (2015). Mammalian target of rapamycin and tuberous sclerosis complex. J. Dermatol. Sci. 79, 93-100. doi: 10.1016/j.jdermsci.2015.04.005

Wu, P. Z., O'Malley, J. T., de Gruttola, V., and Liberman, M. C. (2020). Age-related hearing loss is dominated by damage to inner ear sensory cells, not the cellular battery that powers them. J. Neurosci. 40, 6357-6366. doi: 10.1523/JNEUROSCI.0937-20.2020

Yamasoba, T., Lin, F. R., Someya, S., Kashio, A., Sakamoto, T., and Kondo, K. (2013). Current concepts in age-related hearing loss: epidemiology and mechanistic pathways. Hear. Res, 303, 30-38. doi: 10.1016/j.heares.2013.01.021

Ye, X., Luo, H., Chen, Y., Wu, Q., Xiong, Y., Zhu, J., et al. (2015). MicroRNAs 99b-5p/100-5p regulated by endoplasmic reticulum stress are involved in abeta-induced pathologies. Front. Aging Neurosci. 7:210. doi: 10.3389/fnagi.2015.00210

Zhang, Y., Bokov, A., Gelfond, J., Soto, V., Ikeno, Y., and Hubbard, G. (2014) Rapamycin extends life and health in C57BL/6 mice. J. Gerontol. A Biol. Sci. Med. Sci. 69, 119-130. doi: 10.1093/gerona/glt056

Conflict of Interest: The authors declare that the research was conducted in the absence of any commercial or financial relationships that could be construed as a potential conflict of interest.

Copyright (c) 2021 Altschuler, Kabara, Martin, Kanicki, Stewart, Kohrman and Dolan. This is an open-access article distributed under the terms of the Creative Commons Attribution License (CC BY). The use, distribution or reproduction in other forums is permitted, provided the original author(s) and the copyright owner(s) are credited and that the original publication in this journal is cited, in accordance with accepted academic practice. No use, distribution or reproduction is permitted which does not comply with these terms. 